7th International Workshop on Astronomy and

Relativistic Astrophysics (IWARA 2016)

International Journal of Modern Physics: Conference Series

Vol. 45 (2017) 1760048 (4 pages)

(C) The Author(s)

DOI: $10.1142 / \mathrm{S} 2010194517600485$

\title{
About Bonnor Solution and Gravitational Redshift
}

\author{
Orlenys Troconis, Viviane Alfradique, and Rodrigo Negreiros \\ Departamento de Física \\ Universidade Federal Fluminense (UFF) \\ Av. Gal. Milton Tavares de Souza Gragoatá, Niterói, Rio de Janeiro 24210-346, Brazil \\ troconiso@if.uff.br, vivianeapa@if.uff.br, negreiros@if.uff.br
}

Published 15 August 2017

\begin{abstract}
We consider the analytical Bonnor solution for a relativistic neutron star and discuss about the limit in which this solution satisfies Einstein-Maxwell equations. We study the gravitational redshift for Bonnor solution without electric charge. We find that for stars with magnetic fields up to $10^{16} \mathrm{G}$ in the center, the gravitational redshift for Bonnor metric differs from the Schwarzschild solution on a term that depends on magnetic dipole moment and the polar angle of the emission signal.
\end{abstract}

Keywords: Bonnor Solution, Gravitational Redshift, Neutron Star.

PACS numbers: 26.60.-c

\section{Introduction}

Since the seminar paper by Oppenheimer and Snyder ${ }^{1}$, most of the work dedicated to the problem of general relativistic gravitational collapse, deal with spherically symmetric fluid distribution. In reality, in the study of self gravitating compact objects it is usually assumed that small deviations from spherical symmetry are likely to take place (see Ref. ${ }^{2}$ and references therein). Such small deviations, are not appropriate for stars with strong magnetic fields (whose surface field may be of the order of $\left.B \sim 10^{15} \mathrm{G}\right)^{3}$, where a full axially symmetric treatment is necessary to properly describe the structure of the star ${ }^{4}$, this justify the need to have available exact non-spherical analytical solutions of Einstein equations.

The problem of axially-symmetric solutions for Einstein-Maxwell field equations was considered by Bonnor ${ }^{5}$ in 1966 , he studied a massive source carrying a magnetic dipole moment, but the Schwarzschild solution is no recovered when this parameter is zero. Two years later, Martin and Pritchett ${ }^{6}$ investigated the gravitational field of a source characterized by mass, electric charge and magnetic dipole moment.

This is an Open Access article published by World Scientific Publishing Company. It is distributed under the terms of the Creative Commons Attribution 4.0 (CC-BY) License. Further distribution of this work is permitted, provided the original work is properly cited. 
The solution was written in terms of the parameters, but it is an approximation to second order on gravitational constant of Einstein-Maxwell equations.

In 1987 Gutsunaev and Manko ${ }^{4}$ studied the spacetime outside of a massive source with magnetic dipole moment. This is the exact external analytical solution for this kind of system, but it is not written in terms of the magnetic dipole moment, so the use of this solution is complicated. With the goal to study the dragging of inertial frames effect, in 1991 Bonnor ${ }^{7}$ found the exterior solution of a massive source with a magnetic dipole moment and electric charge without rotation. Bonnor found that dragging of inertial frames, which is an effect normally associated with rotating bodies, can be present in a static massive charged magnetic dipole.

In this work we will discuss about the Bonnor solution found in 1991 but without electric charge (for avoiding the inertial dragging effect) and study the gravitational redshift for neutron stars with magnetic fields up to $10^{16} \mathrm{G}$.

\section{Bonnor Solution}

In 1991 Bonnor considered a static massive charged magnetic dipole, and showed that its gravitational field drags inertial frames, and causes gyroscopes to precess. In spherical coordinates $x^{\mu}=(t, R, \theta, \alpha)$ Bonnor metric is given by

$$
\begin{aligned}
d s^{2}= & \left(1-\frac{2 m}{R}+\frac{e^{2}}{R^{2}}+\frac{\mu^{2} \cos ^{2} \theta}{R^{4}}\right) d t^{2}-\left(1-\frac{2 m}{R}+\frac{e^{2}}{R^{2}}+\frac{\mu^{2} f(\theta)}{R^{4}}\right)^{-1} d R^{2} \\
& -R^{2}\left(1-\frac{\mu^{2} f(\theta)}{R^{4}}\right) d \theta^{2}-R^{2} \sin ^{2} \theta\left(1-\frac{\mu^{2} \cos ^{2} \theta}{R^{4}}\right) d \alpha^{2} \\
& -\frac{2 e \mu \sin ^{2} \theta}{R^{2}} d \alpha d t,
\end{aligned}
$$

where $m, e$ and $\mu$ represents the mass, charge and magnetic dipole moment, respectively and

$$
f(\theta)=\frac{1}{6}\left(\sin ^{4} \theta-6 \sin ^{2} \theta \cos ^{2} \theta+2 \cos ^{4} \theta\right) .
$$

It is important to mention two points about this solution; first point is this solution is an approximation to second order on mass, electric charge and magnetic dipole moment on metric potentials and to first order on electromagnetic potentials of Martin and Pritchett ${ }^{6}$ solution. The second point is that the inhomogeneous Maxwell equation is not satisfyes in this approximation. Therefore, Bonnor 1991 solution should be taken with care. However, two positive points of this solution is the fact it is written in terms of the parameters and when the charge and magnetic dipole moment is zero, Schwarzschild solution is recovered as we can see from Eq. (1).

\subsection{Bonnor Solution Without Electric Charge and Gravitational Redshift}

Gravitational redshift is the process by which electromagnetic radiation originating from a strong gravitational field is reduced in frequency (or energy) when observed 


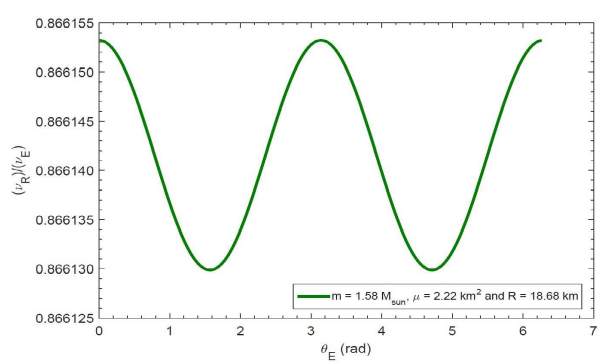

(a) $2 \mathrm{~m} / \mathrm{R}=0.25$

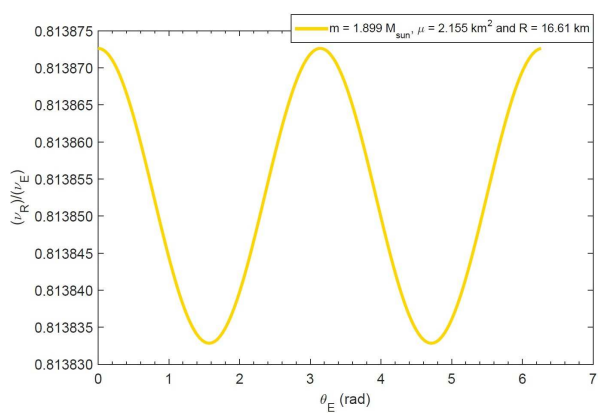

(c) $2 \mathrm{~m} / \mathrm{R}=0.338$

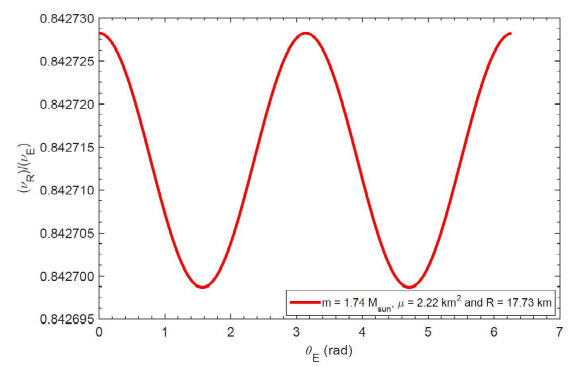

(b) $2 \mathrm{~m} / \mathrm{R}=0.29$

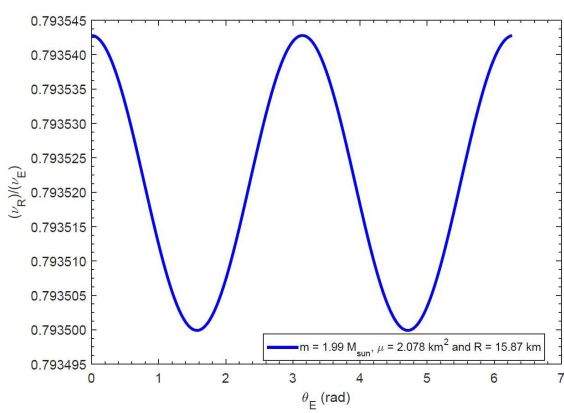

(d) $2 \mathrm{~m} / \mathrm{R}=0.371$

Fig. 1. Variation of the rate between emission and reception frequency as a function of the polar angle of emitted particle for different neutron stars.

in a region so far from the source. The relation between $\nu_{E}$, frequency of emitted signal and $\nu_{R}$, frequency of receives signal is given by ${ }^{8}$

$$
\frac{\nu_{R}}{\nu_{E}}=\sqrt{\frac{g_{00}(E)}{g_{00}(R)}} .
$$

For the line element given by Eq. (1), without electric charge and taken into account the distance between emitted and received particle tends to infinity Eq. (2) goes to

$$
\frac{\nu_{R}}{\nu_{E}}=\sqrt{\left(1-\frac{2 m}{R}+\frac{\mu^{2} \cos ^{2} \theta}{R^{4}}\right)} .
$$

\section{Results and Conclusions}

We study the gravitational redshift for stars with magnetic fields up to $10^{16} \mathrm{G}$ using Eq. (3). 
From the figures below we can conclude that

- When the emitted particle is set at equatorial plane, the particle that receives the signal can not identify if the spacetime generated by this kind of source is described by the Schwarzschild or Bonnor solution.

- For the same value of $\mu$, the redshift effect is bigger for the star with the biggest compactness parameter $(2 \mathrm{~m} / R)$, we can see that in the two first graphics in Fig. 1.

- The Bonnor solution should be taken with care because it is an approximation of Martin \& Pritchett solution, which is itself an approximation.

\section{References}

1. J. Oppenheimer and H. Snyder, Phys. Rev. 56, 455 (1939).

2. L. Herrera, A. Di Prisco, J. Martin, J. Ospino, N. O. Santos, and O. Troconis, Phys. Rev. D 69, 084026 (2004).

3. V. Dexheimer, R. Negreiros, and S. Schramm, Eur. Phys. J., 48, 189 (2012).

4. Ts. I. Manko and V. S. Pritchett, Phys. Lett. A. 123, 215 (1987).

5. W. B. Bonnor, Zeitschrift fur Physik 190, 444 (1966).

6. A. W. Martin and P. L. Pritchett, J. Math. Phys. 9, 593 (1968).

7. W. B. Bonnor, Phys. Lett. A. 158, 23 (1991).

8. M. P. Hobson, G. Efstathiou, and A. N. Lasenby, General Relativity: an introduction for physicist, 2nd edn. (Cambridge University, 2006). 